\title{
LA VILLE DE CUIVRE, UNE VILLE D’AL-ANDALUS
}

Por

Edgar WEBER

\section{Attestation}

Le conte de la Ville de Cuivre ${ }^{1}$ occupe les Nuits 566-578 dans l'édition de Calcutta (1832-1842). C'est cette numérotation que suit l'édition du P. Salahâni ${ }^{2}$ qui porte le titre suivant : histoire des vases de Salomon (hikâyat al-qamâqim al-sulaymâniyya) ne faisant pas apparaître le titre de la Ville de Cuivre qui y est pourtant mentionnée. L'édition populaire d'al-maktabat al-thaqâfizya ${ }^{3}$ sépare nettement les deux parties. Ainsi les Nuits 559-566 contiennent l'histoire des djinns et des démons emprisonnés dans les vases depuis le règne de Salomon (hikâyat fi sh'ani l-jinn wa l-shayâtîn al-masjûnîna fî̀ lqamâqim min ${ }^{\circ}$ ahdi Sulaymân) et les Nuits 566-572 rapportent l'histoire de la Ville de Cuivre (madinat al-nuhâs). Nous nous reporterons principalement à cette édition dans notre étude.

La traduction de Mardrus ${ }^{4}$ réunit ces deux récits sous le titre d'Histoire prodigieuse de la Ville d'Airain.

Pourquoi mentionner ces légères variantes ? C'est que d'emblée les différentes éditions posent un problème de contenu. Pour les uns, il s'agit d'une seule histoire, pour d'autres il est question de deux histoires, voire davantage. Cette hésitation dans les éditions confirme l'idée que la Ville de Cuivre fut en effet originellement un récit

\footnotetext{
${ }^{1}$ En espagnol, ce sujet a été présenté par Maria Jesus Rubiera, dans le chapitre : "la ciudad de cobre" de son livre : La arquitectura en la literatura arabe, Madrid, 1981, pp. 63-68.

${ }^{2}$ Salahâni, alf layla wa layla, Beyrouth, 1957, vol. IV, pp. 237-252 et vol. V, pp. 1-7.

3 Al-maktaba 1-thaqâfiyya, Beyrouth, 1979, vol. III, pp. 203-229.

${ }^{4}$ Mardrus, Paris, Laffont, 1980, vol. I, pp. 781-796 ; N. 339-347.
} 
indépendant ${ }^{1}$ qui fut inséré plus tard dans un ensemble plus large où le merveilleux abonde. Nous pouvons en effet rapprocher ce récit de nombreuses attestations que l'on trouve surtout chez les géographes arabes, après en avoir donné la structure principale.

\section{Résumé du conte}

Le récit de la Ville de Cuivre n'est pas seulement un conte des Mille et Une Nuits, ni seulement un conte des Cent et Une Nuits, mais une légende qui remonte bien avant la constitution définitive des Nuits, puisqu'on le retrouve dans un manuscrit anonyme du IXe siècle, puis chez plusieurs géographes et cosmographes renommés de la civilisation arabo-musulmane.

Dans son expression la plus simple, l'histoire de la Ville de Cuivre est liée au désir d'un souverain umayyade : Abd al-Malik Ibn Marwân qui veut récupérer les vases dans lesquels Salomon a jadis enfermé les djinns rebelles, selon une tradition qui venait de lui être rapporté. Il charge immédiatement Tâhir Ibn Sahl, un grand voyageur du royaume, d'entreprendre cette expédition. Celui-ci se fait aider par un autre grand voyageur: Abd al-Samad, recommandé par Abd alAzîz, le frère d'Abd al-Malik qui gouverne en Egypte. Tous les deux vont trouver Musa Ibn Nusayr au Maghreb pour exécuter le désir du Calife de Damas, car ces vases se trouvent dans cette partie du monde musulman. Durant leur expédition, les trois hommes et leur troupe tombent sur un château immense renfermant le tombeau de Shaddâd, un cavalier de cuivre fixé sur un socle, un djinn attaché au tronc d'un palmier, enfin la Ville de Cuivre où les trois voyageurs réussissent finalement à pénétrer. Tâhir Ibn Sahl y meurt en voulant emmener la jeune fille qui dormait sur un lit richement dressé au milieu d'un palais. Mûsa décide alors de continuer le chemin, non sans avoir chargé les chameaux de toutes les richesses possibles avant d'atteindre finalement le bord de la mer où des pêcheurs noirs retirent

${ }^{1}$ Tarchouna, Mahmoud, Les Marginaux, (Appendice : les marginaux dans les Mille et Une Nuits], Tunis, Imprimeric Officielle, 1982, p. 475. 
de l'eau les vases scellés du temps de Salomon. Puis c'est le retour à Damas auprès du Calife Abd al-Malik.

Ce récit, on s'en doute, connaît de nombreuses variantes selon la tradition qui le relate. Il est intéressant de suivre ces modifications pour voir la genèse et l'évolution du récit.

\section{La Ville de Cuivre dans la tradition géographique}

La version la plus ancienne de la Ville de Cuivre se trouve dans un manuscrit anonyme conservée à la bibliothèque d'Oxford : ta'rîkh Ibn Habîb, daté de la fin du IXe siècle. Mûsa, le célèbre conquérant du Maghreb et de l'Espagne découvre une forteresse en cuivre. Ceux de ses hommes qui réussissent à escalader la muraille en sont renvoyés et demeurent incapables de se souvenir de ce qu'ils ont vu. Mûsa lève le camp sans avoir pu y pénétrer. Le thème de l'inviolabilité reviendra comme une donnée essentielle dans toutes les attestations antérieures à celle des Mille et Une Nuits. Là, Mûsa réussit à pénétrer dans la ville, alors que la tradition classique des géographes le fait échouer chaque fois qu'il entreprend d'y entrer.

A ce thème de l'inviolabilité, Ibn al-Faqîh (m. ap. 903) ajoute celui de la pêche des vases de Salomon ${ }^{1}$. Ainsi le conte de la Ville de Cuivre se trouve-t-il en fait composé de deux récits originairement indépendants, l'un appartenant à ce que l'on pourrait appeler le cycle de Salomon cher à la tradition sémitique judéo-chrétienne, l'autre appartenant au cycle des conquêtes arabes et plus précisément des hauts faits de Mûsa Ibn Nusayr conquérant de l'Afrique du Nord. Selon Ibn al-Faqîh, c'est Alexandre (Dhu l-Qarnayn) qui aurait fait construire la ville pour y déposer ses trésors et l'intérieur aurait été édifié avec la pierre de $b a h t^{\mathcal{Z}}$ dont la propriété est d'attirer, comme

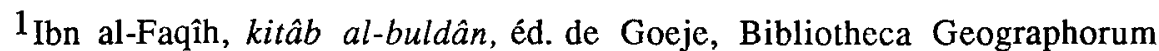
Arabicorum (B.G.A.), tome V, Leyde, 1885, p. 84.

${ }^{2}$ Au chapitre IX : les pays de la légendes, Miquel écrit à propos de la pierre : "Car voici maintenant, chargée de propriétés magnétiques et hallucinatoires, la pierre de l'aigle ou aétie (baht), qui tient du fer par sa composition, mais du cuivre par sa 
l'aimant le fer, les hommes qui la regardent, après avoir provoqué chez eux un irrésistible rire.

Plus proche d'Ibn al-Faqîh dans le temps, $\operatorname{Mas}^{\circ} \hat{u}$ di (m. 956) mentionne deux fois la Ville de Cuivre dans ses célèbres murûj aldhahab (Prairies d'or). Le premier texte situe la ville au Maghreb. "Ensuite vient le pays du Sûs al-Adna qui est distant de Kairouan d'environ 2.300 milles et d'une vingtaine de journées de marche du Sûs al-Aqsa, sur un parcours constamment fertile et cultivé ; [mais au-delà de ce dernier point] on arrive au Wâdi r-raml et au Château Noir, puis au désert de sable dans lesquels se trouve la ville connue sous le nom de Ville de cuivre et Coupoles de Plomb. C'est à cet endroit que se rendit Mûsa $b$. Nusayr du temps de Abd al-Malik b. Marwân et qu'il y vit toutes les merveilles dont il a donné la description dans un livre que l'on se passe de main en main. D'autres disent que [cette ville] se trouvait dans les déserts qui avoisinent l'Espagne et que l'on appelle la Grande Terre"1.

Dans le second passage, $\operatorname{Mas}^{\circ}$ ûdi fait allusion à un livre : akhbâr al-zamân, aujourd'hui perdu, dans lequel il aurait développé l'histoire de cette ville. Il ajoute cependant : "[il a été question...] des sept édifices de l'Espagne, de la ville d'airain (madînat al-sufr) et de la coupole de plomb qui se trouvent dans les déserts de ce pays; [nous avons raconté les aventures] de ses anciens rois, [et dit] qu'il était impossible d'y accéder ; puis [nous avons expliqué] comment elle fut assiégée par le général d'Abd al-Malik b. Marwân, comment les Musulmans furent précipités du haut des murs, en montant à l'assaut, et comment, d'après leur propre récit, ils goûtèrent ensuite les délices de ce monde et de l'autre. [Nous avons mentionné] une autre ville entourée de remparts de cuivre et située sur la côte de l'océan indien, à l'extrémité des déserts de l'Inde; nous avons parlé des aventures des rois de l'Inde dans cette ville, où il leur était impossible de

par sa couleur ; comme le cuivre à Rome, elle donne corps et nom à toute une ville : la madînat al-baht, cheval de bataille de l'encyvlopédie des merveilles, où Ibn al-Faqîh se montre une fois de plus imbattable" : Miquel, La Géographie humaine du monde musulman..., vol. II, Paris, Mouton, p. 491.

${ }^{1}$ Mas $^{\circ}$ ûdi, Les Prairies d'or, trad. Charles Pellat, Paris, CNRS, 1962, p. 149. 
pénétrer ; enfin des [eaux] qui sortent du Wâdi r-raml et de se dirigent vers cette cité"1.

La description de Mas $^{\circ}$ ûdi contrairement au récit du ta'rîkh Ibn Habîb, omet de mentionner la pierre baht qui fait rire. Mais en accord avec Ibn al-Faqîh, il semble attester que le récit de la Ville de Cuivre faisait l'objet d'un livre à part.

Un contemporain de Mas $^{\circ}$ ûdi qui lui aussi évoque la Ville de Cuivre est le grand historien Tabari (838-923) auteur d'un monumental ouvrage d'histoire dont il ne reste que le résumé en treize volumes! Tabari enregistre très souvent des données sans les harmoniser entre elles, à la manière en quelque sorte de la compilation du hadîth. Mais il est plus intéressant encore de constater que le récit sur la ville magique vient à la suite d'une explication sur la fontaine d'airain dont parle le Coran : 34, 11 : " A Salomon [Nous soumîmes] le vent... Pour lui, Nous fîmes couler la Source d'Airain. Parmi les Djinns, il en était qui travaillaient à sa discrétion, avec la permission d'Allah... Pour lui, ils faisaient ce qu'ils voulaient : des sanctuaires, des statues, des chaudrons [grands] comme des bassins et des marmites stables".

Tabari explique donc qu'avec cet airain qui coulait, Salomon fit construire cette fameuse ville dans un endroit où les hommes ne passent pas : "Salomon réunit les péris et les devs, ainsi que les hommes et il leur demanda de lui construire avec [cet] airain coulant un monument qui subsistât jusqu'au jour du jugement... " pour "faire de cette ville le dépôt de tous les trésors et de tous les livres que tu (Salomon) as en ton pouvoir"2. Cette ville retirée du monde et où aucun homme n'a jamais pu se rendre sauf Salomon ou Alexandre selon les traditions, est découverte un jour par par Mûsa Ibn Nusayr du temps d'Abd al-Malik Ibn Marwân. Les murailles étaient tellement hautes qu'ils entassèrent tous les bâts des chameaux pour atteindre les créneaux. Le premier qui y monta "fit un visage riant à ses compagnons, se mit à rire aux éclats, se précipita de l'autre côté des murailles et disparut. Personne de tous ceux qui étaient présents

\footnotetext{
${ }^{1}$ Mas $^{\circ}$ ûdi, Les Prairies d'or,trad. Charles Pellat, tome II, CNRS, Paris, 1965, p. 547.

${ }^{2}$ Tabari, De la création à David, trad. Zotenberg, Paris, Sindbad, 1984, p.53.
} 
n'entendit plus parler de lui"1. Ainsi moururent trois compagnons. Mûsa décida d'abandonner le projet d'entrer dans cette ville. En faisant le tour des murs, ils découvrirent une inscription en vers sur le mur rappelant que le destin de l'homme est de mourir. Le texte des Mille et Une Nuits multipliera ce genre d'inscription. Puis Tabari passe à l'histoire de Shaddâd fils de Ad. Ce rapprochement n'est pas gratuit non plus si l'on observe que les Mille et Une Nuits mentionnent également Shaddâd lorsque Mûsa découvre un immense château dans lequel se trouve sa tombe. On a limpression que Tabari fait converger vers un centre des récits originellement indépendants.

Yâqût (1179-1229) dans son $m u^{\circ}$ jam al-buldân attribue à Salomon la construction de la Ville de Cuivre pour y mettre ses trésors, comme l'atteste également la version de Tabari. Yâqût reproduit un texte d'Ibn al-Faqîh, qui, lui-même, se retrouve chez Qazwîni. La trtaduction est la suivante.

"La Ville de Cuivre : on dit aussi madinat al-sufr. Son histoire est difficile à croire à cause de sa divergence avec ce qui est habituel. Je ne m'en porte pas responsable. Je n'écris que ce que j'ai trouvé dans les livres célebres que les savants ont composé\&s. [Quoi qu'il en soit], c'est une ville connue, aussi l'ai-je mentionnée à mon tour. Ibn al-Faqîh rapporte: Parmi les villes d'al-Andalus, il y a la madînat alsufr. Certains savants pensent qu'elle a été construite par Dhu lQarnayn qui y a déposé ses trésors et sa science et en [interdit] l'entrée avec des talismans (tilasm). Personne ne l'a encore découverte. Il construisit l'intérieur avec la pierre baht ${ }^{2}$ qui est [comme] un aimant pour les hommes ${ }^{3}$. En effet, quand un homme la regarde, il ne peut plus s'empêcher de rire, ni de se jeter vers elle. Il

\footnotetext{
1Tabari, op. cit., p. 54.

Le texte arabe donne al-bahta (au féminin).

${ }^{3}$ On trouve la mention de la pierre baht chez Zuhri au XIIe siècle dans son kitâb al-jughrâfiyya. Celui qui la regarde demeure stupéfait (bahita) et ne peut plus parler. Zuhri mentionne dans le même ouvrage l'existence d'un château, al-qasr almushayyad dont l'intérieur aurait été construit avec cette pierre rapportée par Alexandre du pays des Zanj. Sa présence explique pourquoi les hommes qui veulent escalader cet immense château sont attirés vers l'intéricur et disparaissent sans laisser de trace. cf. Zuhri, kitâb al-jughrâfiyya, éd. Hadj-Sadok, Damas, Bulletin d'Etudes Orientales, tome XXI, p. 186 et pp. 266-267.
} 
ne peut plus s'en détacher non plus jusqu'à ce qu'il meure. [Cette ville] se situe dans les déserts (mafâwiz) d'al-Andalus. Lorsqu'Abd al-Malik Ibn Marwân en entendit parler ainsi que de ses trésor et de la science qu'elle renfermait et [qu'il apprit] qu'à côté d'elle se trouvait un lac renfermant également d'immenses trésors, il écrivit à Mûsa Ibn Nusayr son lieutenant au Maghreb et lui donna l'ordre de s'y rendre et de tout faire pour y pénétrer, puis de lui faire savoir ce qu'elle renferme. Il remit la lettre à Tâlib Ibn Mudrik qui s'en chargea et se mit en route et arriva auprès de Mûsa Ibn Nusayr à Kairouan. Dès qu'il lui remet la lettre, Musa équipa mille cavaliers et partit en direction de la ville. Quand il en revint, il écrivit à Abd al-Malik Ibn Marwân : Au nom du Dieu Miséricordieux et Clément, que Dieu se montre bon envers le Prince des Croyants et qu'il lui procure le bien dans ce monde et dans l'autre. O Prince des Croyants, je t'informe que je me suis équipé pour quatre mois et suis parti dans les déserts $d^{\prime}$ al-Andalus accompagné de mille cavaliers. Je me suis enfoncé dans des chemins dont les traces étaient perdues et dont les points d'eau avaient disparu. [Depuis longtemps] on n'en avait plus ni trace ni nouvelle. [Je suis donc parti] pour trouver la construction d'une ville dont personne n'avait jamais $v u$ de semblable, $n i$ rien entendu de comparable. J'ai marché quarante trois jours, quand soudain, nous apparut l'éclat de sa grandeur, à une distance de cinq jours de marche? Son aspect terrible nous effraya et remplit nos coeurs d'épouvante [tellement elle était] immense et la contrée lointaine. Quand nous fûmes près d'elle, elle était merveilleuse et son aspect terrible comme si elle n'avait pas été faite de main d'homme. Je descendis à son angle oriental et fis la prière du soir avec mes compagnons. Nous passâmes la nuit la plus horrible que Musulmans aient jamais passée. A notre réveil, nous proclamâmes la grandeur de Dieu, ravis et contents d'être au matin. Puis, j'ai donné l'ordre à un de mes compagnons à la tête de cent cavaliers de faire le tour de la muraille. Ils partirent deux jours et revinrent au matin du troisième m'informant qu'il n'y avait pas de porte et qu'aucun chemin n'y conduisait.. Je fis alors rassembler tous les bagages de mes compagnons au pied de la muraille et les fit entasser les uns sur les autres pour voir si quelqu'un pouvait y monter et me rapporter ce qui se trouvait à l'intérieur. Mais nos bagages n'atteignirent même pas le quart de la hauteur de la muraille. Je donnai alors l'ordre de prendre des échelles (salâlim). Ce qui fut fait. Je les fis fixer les unes aux 
autres avec des cordes et les fis dresser contre le mur, promettant mille dirhams à celui qui me renseignerait sur la ville. Un de mes compagnons avança et escalada l'échelle, implorant Dieu et récitant des versets du Coran. Quand il fut arrivé au faîte de la muraille et qu'il surplomba ce qui s'y trouvait, il éclata de rire et y s'élança dans la ville. Nous lui criames: Dis-nous ce que tu as, et ce que tu vois. Mais il ne nous répondit pas. Je promis alors mille dinars a celui qui y monterait et qui me renseignerait sur la ville et cet homme. Un homme de Himyar se présenta, prit les dinars et les déposa dans ses bagages (rahl), puis il monta. Quand il fut au sommet de la muraille, il éclata de rire et s'élança à son tour à l'intérieur. Nous lui criâmes également : Dis-nous ce qu'il y a derrière toi, dis-nous ce que tu vois! Mais il ne nous répondit pas. Un troisieme y monta, mais il en fut de lui comme des deux précédents. [Finalement] plus personne ne voulut y monter, chacun ayant pitié de lui-même. Je perdis tout espoir de voir quelqu'un y monter et ne pus plus prétendre à en savoir plus, aussi je partis en direction du lac en longeant la muraille et j'aboutis à un endroit où était gravée une inscription en langue himyarite que je fis recopier"1.

Le contenu de l'inscription, en dix vers, mentionne l'impuissance de l'homme et son destin mortel ainsi que la construction de la Ville par Salomon. Mais l'ordre donné aux djinns d'exécuter l'ouvrage, est en contradiction avec le texte plus haut où le constructeur est Dhu 1-Qarnayn (Alexandre) et non plus Salomon.

Ici se termine la description proprement dite de la Ville de Cuivre, mais Yâqût consacre encore une page à la description du lac dans lequel apparaît un djinn dont le père a été enfermé par Salomon. Mûsa interroge le djinn sur sa présence. Il lui répond qu'il avait entendu du bruit et cru que c'était le retour d'al-Khidr. Car al-Khidr avait l'habitude de revenir une fois l'an sur le lac. Puis le texte mentionne la pêche de vases (hubâb) de cuivre scellés avec du plomb d'un desquels s'échappait un cavalier en cuivre tenant dans sa main une lance en cuivre. Quand Abd al-Malik eut finit de lire la lettre de Mûsa, il en demanda le sens à un certain al-Zuhri qui lui confïma que les hommes qui se jetaient en bas de la ville, le faisaient parce que des djinns se trouvaient à l'intérieur de la ville ; par ailleurs, la

1Yâqût, mu jam al-buldân, Dâr Beyrût, Beyrouth, vol. 5, 1957, pp. 80-83. 
fumée qui sortait des vases repêchés par les Noirs était des djinns que Salomon y avait enfermés.

Le thème du lac manque chez certains géographes, mais le cavalier de cuivre, l'inscription ou les inscriptions et la pêche des vases renfermant des djinns sont généralement reproduits, mais dans un ordre différent. Dans les Mille et Une Nuits, les vases sont la raison première du voyage et le cavalier en cuivre revient deux fois, une fois comme statue indiquant la direction à suivre, une seconde fois sous la forme d'un sculpture ouvrant la tour sur la muraille qui permettait de descendre dans la ville. Ce merveilleux se retrouve dans un autre texte majeur que nous reproduisons maintenant.

Abu Hâmid al-Gharnâti (m. 1170) qui excelle dans l'art de la rihla agrémentée de récits merveilleux et de légendes mentionne que c'est bien Salomon qui a fait élever la ville. Il ne mentionne plus la pierre baht, bien que les hommes qui escaladent la muraille se mettent à rire avant de se jeter en bas. Le récit est un des plus riches en imagination et mérite d'être reproduit en entier.

"Récit de la Ville de Cuivre, construite par les djinns pour Salomon fils de David (sur eux soit la paix) dans les déserts (fayâfi) d'al-Andalus au Maghreb al-Aqsâ, non loin (qarîban) de la mer des Ténèbres (bahr al-zulumât). Lahqal Ibn Ziyâd rapporta qu'Abd alMalik entendit rapporter [un jour] l'histoire (khabar) de la Ville de Cuivre qui se trouve en al-Andalus. Il écrivit [aussitôt] à son gouverneur ( ${ }^{\circ}$ âmil) du Maghreb : J'ai appris l'histoire de la Ville de Cuivre construite par les djinns pour Salomon fils de David (sur eux soit la paix); vas-la voir et décris-moi les merveilles que tu auras vues de tes propres yeux. Réponds-moi vite, si Dieu le veut. Lorsque le gouverneur du Maghreb reçut la lettre d'Abd al-Malik, il sortit avec une immense armée, un important équipement et des vivres pour un bout de temps, [accompagné] de guides (adillâ') [capables] de les conduire à cette ville. [Mûsa] marcha sur une route peu commune pendant quarante jours avant de déboucher sur une vaste terre avec beaucoup d'eau, de sources, d'arbres, de bêtes sauvages, d'oiseaux, d'herbes et de fleurs. La muraille de la Ville de Cuivre leur apparut comme si elle n'avait pas été faite de main d'homme. Ils en étaient effrayés. Puis l'émir Mûsa Ibn Nusayr divisa son armée en deux parties et installa chacune d'elle à chaque côté de la muraille de la Ville. Il envoya [également] un général à la tête de mille cavaliers 
faire le tour de la Ville pour voir s'il y découvrirait une porte et s'il rencontrerait près d'elle âme qui vive. Le général partit et s'absenta six jours. Le septième jour il revint avec ses hommes et rapporta qu'il avait mis six jours pour faire le tour de la ville sans apercevoir âme qui vive, sans trouver [la moindre] porte. Mûsa Ibn Nusayr dit alors : comment [faire pour] connaître ce qu'il y a à l'intérieur de cette ville? Les ingénieurs (muhandisûn) lui répondirent : Donne l'ordre de creuser sous ses fondations. Peut-être pourras-tu pénétrer à l'intérieur. [C'est ce qu'il fit]. Ils creusèrent alors à la base de la muraille de la ville jusqu'à ce qu'ils atteignirent [un niveau] d'eau. Les fondations du cuivre étaient si profondément enfoncées sous terre qu'ils furent arrêtés par l'eau (ghalabahum al-mâ'). Ils comprirent alors qu'ils ne pourraient jamais entrer par les fondations. Les ingénieurs lui conseillèrent alors : Fais élever, près d'un angle d'une des tours de la ville, une construction qui [nous permette] de surplomber la ville. Il [en] donna l'ordre et ils [se mirent à] découper des pierres et à préparer du plâtre et de la chaux vive. Ils éleverent à côté de la ville dans un angle d'une tour, une construction d'une hauteur de trois cents coudées, au point de ne plus pouvoir ajouter une pierre de plus, ni du plâtre ni de la chaux vive. Il manqua encore deux cents coudées [avant d'atteindre] la muraille. Mûsa Ibn Nusayr donna alors l'ordre d'employer du bois [et de l'ajouter] à la construction en pierres. Ils arriverent ainsi à cent soixante-dix coudées de plus. Ils fabriquèrent alors une immense échelle qu'ils y montèrent au moyen de cordes et qu'ils appuyèrent sur le sommet du mur. Puis, Mûsa Ibn Nusayr fit venir un héraut [pour] annoncer aux hommes: A celui qui grimpera au sommet de la muraille de la ville, je donnerai son prix du sang. [Aussitôt] un valeureux s'avança parmi ses hommes et réclama le prix du sang. Mûsa Ibn Nusayr ordonna de le lui remettre. Il le prit et le laissa à l'émir en dépôt, disant : Si j'en sors vivant, ce sera mon salaire et je le prendrai, mais si je meurs, $j l$ reviendra à mes héritiers comme [une part] d'héritage. Puis, il grimpa jusqu'au sommet de la muraille de la ville et qu'il surplomba cette dernière, il se mit à rire, à battre des mains et à se jeter à l'intérieur de la ville. [Les compagnons] entendirent alors un immense vacarme et des cris terribles. Ils [en] furent grandement effrayés. Ces cris durèrent trois jours et trois nuits, avant de cesser. [Les hommes de Mûsa] appelèrent alors leur compagnon, mais sans obtenir de réponse. Quand ils eurent perdu tout espoir [de le retrouver], [Mûsa] 
fit venir un autre héraut [pour lui faire annoncer] : L'émir accordera mille dinars à celui qui grimpera sur le sommet de la muraille. [Aussitôt], un autre valeureux se présenta en disant : Je grimperai au sommet. L'émir ordonna de lui remettre [sur le champ] les mille dinars. Il les prit et en fit la même chose que son prédécesseur. L'émir lui donna ce conseil : Ne fais pas comme [ton compagnon], informe-nous de ce que tu vois et ne descends pas chez les autres, en abandonnant tes compagnons. Il leur en fit la promesse. Mais quand il arriva au sommet de la ville, il se mit à rire, à battre des mains et à se jeter [en bas]... Toute l'armée se met alors à crier : Ne fais pas cela. [Mais] il ne les écouta pas et disparut. Ils entendirent une nouvelle fois des cris terribles, encore plus forts que les premiers, au. point qu'ils craignirent [maintenant] pour leur vie. Ces cris durèrent trois jours et trois nuits avant de cesser. Mûsa Ibn Nusayr dit alors: [Si] nous partons d'ici sans rien savoir de cette ville, que vais-je écrire et répondre au prince des croyants? Il ajouta : Celui qui [y] grimpera, je lui ferai remettre deux fois le prix du sang. Un troisième homme se présenta en disant: $J^{\prime} y$ monte, mais attachez-moi à la taille une forte corde et tenez en le bout. Si je me jette en bas, vous m'en empêchez. C'est ce qu'ils firent. L'homme grimpa alors au sommet et une fois arrivé, il se mit à rire et [voulut] se jeter en bas. [Aussitôt], ils tirèrent sur la corde mais l'homme tira vers la ville [si fort] que son corps se rompit en deux. La moitié inférieure avec les cuisses et les jambes tomberent d'un côté et l'autre moitié tombée à l'intérieur de la ville. Les cris et le vacarme redoublèrent de plus belle. L'émir désespéra de connaître quelque chose sur la ville, disant que la dans la ville [il devait y avoir] des djinns qui s'emparent de tous ceux qui veulent descendre dans la ville. L'émir Mûsa donna alors l'ordre à l'armée de partir. Ils avaient dépassé la ville d'une parasange environ, lorsque [Mûsa] aperçut des plaques (alwâhan) de marbre blanc. Chaque plaque avait environ vingt coudées et sur chacune était gravés des noms de rois, de prophètes, de pharaons, de Chosroès, de tyrans, des conseils et des mises en gardes, le souvenir du Prophète Mahomet et de sa Communauté et leur glorieuse [renommée], ainsi que [la place] prestigieuse qu'ils occupent auprès de Dieu. [Or], il y avait parmi les savants qui accompagnaient [Mûsa], quelqu'un qui savait lire toutes les langues. Ils retranscrirent le contenu de toutes les plaques. Soudain, ils aperçurent au loin une statue (sûra) en cuivre. Ils se dirigèrent vers elle et trouverent [en 
effet] une statue d'un homme tenant dans sa main une plaque en cuivre sur laquelle était gravé [ce texte] : N'allez pas plus loin, revenez sur vos pas, n'entrez pas sur cette terre, [sinon] nous mourrez. Mûsa prit la parole : Sur une terre si blanche, avec autant d'arbres, de plantes et d'eau, comment pourrait-on y mourir? Il donna alors l'ordre a quelques-uns de ses esclaves d'avancer sur cette terre. [Aussitôt] des fourmis grosses comme des bêtes sauvages, bondissant parmi les arbres, mirent en pieces ces hommes ainsi que leurs chevaux et se dirigèrent vers l'armée comme un gros nuage, ne s'arrêtant que lorsqu'ils atteingnirent la statue. Ils n'allerent pas plus loin. [Mûsa et ses hommes] en étaient étonnés. Ils s'éloignèrent de la ville, marchant en direction de l'Orient, jusqu'd ce qu'ils virent de nouveau beaucoup d'arbres.

Ce passage sera également repris par le fameux cosmographe Qazwîni (1203-1283), dans le kitâb athâr al-buldân ${ }^{1}$ mais, en ne mentionnant que le passage sur la ville, sans l'épisode des vases de Salomon.

En revanche l'Abrégé des merveilles du XIV-XVe s. donne une nouvelle variante sur l'emplacement de cette ville merveilleuse. "[Le roi Sâ] réunit tous les ouvriers d'Egypte et il construisit dans le Wâh el-Aksa (l'oasis extrême), une ville dont les murailles avaient cinquante coudées de haut, et il y déposa beaucoup de secrets des sciences et de grands trésors. Cette ville est celle à laquelle arriva Mûsa, fils de Nosaïr, au temps des Omeyades, après qu'il eût été investi de l'autorité dans le Maghreb. Ce personnage étant venu en Egypte se dirigea au moyen des étoiles sur le Wâh el-Aksa ... et il arriva devant une ville fortifiée, ayant des portes de fer. Il voulut ouvrir l'une de ces portes, mais il ne le put pas, parce que le sable s'était amoncelé tout autour d'elle. Ils fit monter des hommes qui sautèrent à l'intérieur, ne sachant pas comment ni sur quoi ils retomberaient, ni ce qu'ils rencontraient. Mais n'ayant trouvé aucun moyen d'entrer, il abandonna cette ville et passa... On dit que personne avant ou après lui ait pénétré dans cette ville" 2 . Remarquons que l'auteur de cette recension, (pour certains Ibn Wâsif

${ }^{1}$ Qazwîni, athâr al-buldân, éd. Wüstenfeld, tome, III, Göttingen, 1848, p. 375.

$2_{2}$ 'Aberégé des Merveilles, trad. Carra de Vaux, Paris, Sindbad, 1984, p.119. 
Shâh ou un autre) ne donne pas le nom de la ville. Pour lui, c'est une ville anonyme que Mûsa ne réussit pas à prendre.

Maqrîzi (1364-1442), qui copie l'Abrégé des Merveilles dans son khitat où il compile tout ce que l'on sait sur l'Egypte situe l'existence de la ville au sud du haut Nil.

Ibn Khaldûn (1332-1406), un contemporain, met en revanche, sérieusement en doute l'existence d'une telle ville dans le cadre de sa réflexion sur l'histoire des faits possibles ou impossibles : "Il y a aussi Al-Mas'oudi et sa fameuse Ville de Cuivre (madînat al-nuhâs) qui aurait été située dans le désert de Sijilmâsa, traversé (au début du VIIIe siècle) par Mûsa b. Nusayr, au cours de son expédition au Maghreb. On dit que les portes de cette ville étaient fermées. Celui qui veut entrer en escaladant les murs, quand il arrive au faîte des remparts, bat des mains, se jette dans le vide et disparaît. C'est un conte digne des des Mille et Une Nuits. Guides et voyageurs ont parcouru le désert de Sijilmâsa : ils n'ont jamais rien vu de semblable. Tous les détails ci-dessus sont invraisemblables. Il y a juste assez de métaux pour les vases et les ustensiles. il est absurde de penser qu'on pourrait disposer d'une quantité (de cuivre) suffisante pour en construire une ville"1. Ibn Khaldûn semble faire la critique interne des textes, pour s'assurer de leur objectivité. Le récit de la Ville de Cuivre allait devenir un conte des Mille et Une Nuits et ainsi se détacher définitivement des récits véridiques que recherche Ibn Khaldûn. En effet, pour lui, "lorsqu'il s'agit d'événements matériels, il faut reconnaître, avant tout, leur conformité (avec la réalité), c'est-à-dire se demander s'ils sont possibles" 2 . Ibn Khaldûn a conscience de poser un vrai problème à l'historien. "Notre propos actuel est d'une conception nouvelle, d'une grande originalité et d'une extrême utilité "(idem).

Au terme de cette revue des principales mentions de la Ville de Cuivre dressons un tableau récapitulatif des principales caractéristiques de cette ville. La première colonne indique les données d'Abu Hâmid al-Gharnâti, la seconde : Tabari, la troisième :

${ }^{1}$ Ibn Khaldûn, Discours sur l'Histoire universelle, al- muqaddima, trad. V. Monteil, Paris, Sindbad, 1968, tome I, pp. 73-74.

2 Ibn Khaldûn, op. cit. p. 74. 
L'Abrégé des merveilles, la quatrième : Mardrus, la cinquième : les Cents et Une Nuits, et la sixième : Yâqût, $m u^{\circ}$ jam al-buldân.

\begin{tabular}{|c|c|c|c|c|c|c|}
\hline nom & cuivre & --- & - & airain & cuivre & sufr \\
\hline auteur & djinns & djinns & Sâ & -- & - & djinns \\
\hline destinataire & Salomon & Salomon & Sâ & - & - & Alex. \\
\hline lieu & Andalus & Andalus & Egypte & - & -- & Andal. \\
\hline chef & Malik & Malik & -- & Malik & Malik & Malik \\
\hline exécuteur & Mûsa & Mûsa & Mûsa & les 3 & les 3 & Mûsa \\
\hline adjuvants & guides & troupe & troupe & $\mathrm{T}+\mathrm{S}$ & $\mathrm{T}+\mathrm{S}$ & Tâlib \\
\hline durée & $40 \mathrm{j}$. & $40 \mathrm{j}$ & $7 \mathrm{j}$ & $\cdots$ & $40 \mathrm{j}$. & 4 mois \\
\hline cadre & végét. & désert & sable & silence & désert & déserts \\
\hline tour & $6 \mathrm{j}$. & -- & -- & heures & jours & $2 \mathrm{j}$. \\
\hline essai & sape & -- & -- & - & - & -- \\
\hline moyen & échelle & selles & - & échelle & échelle & échelle \\
\hline portes & --- & -- & fer & -- & 25 & -- \\
\hline tentatatives & 3 & 3 & plus.. & 1 & 5 & 3 \\
\hline bruit & $3 \mathrm{j}$. & - & - & -- & - & -- \\
\hline rire & hom. & hom. & -- & -- & - & hom. \\
\hline réussite & -- & -- & - & Mûsa & A.Sam. & --- \\
\hline inscription & oui & -- & -- & 4 & 7 & 1 \\
\hline cavalier & oui & -- & -- & oui & oui & oui \\
\hline châtiment & fourmis & - & - & - & - & $\cdots$ \\
\hline mort Tâhir & $\ldots$ & - & - & oui & oui & -- \\
\hline
\end{tabular}

$\mathrm{T}+\mathrm{S}$ représente Tâhir (alias Tâlib) et Abd al-Samad; Sâ est un pharaon d'Egypte ; A.Sam est Abd al-Samad ; les 3 sont : Mûsa Ibn Nusayr, Tâhir et Abd al-Samad.

\section{Le merveilleux dans la tradition arabe}

Le récit de la Ville de Cuivre ne doit pas être considéré comme un pur fruit de l'imagination humaine, comme on l'entendrait en Occident. Car dans la tradition arabo-musulmane l'imaginaire ne recoupe pas exactement l'imaginaire occidental. Les djinns par exemple, que l'Occidental aurait tort de ranger du côté des fées, sont 
des créatures de Dieu dont l'existence ne peut être mise en doute parce que le Coran les authentifie. Le Coran certifie aussi l'existence de Salomon et sa puissance sur les mêmes djinns. Il devient donc tout naturellement pensable et plausible, pour un homme appartenant à cette culture arabo-musulmane, de croire que l'emprisonnement des djinns dans des vases est un des exemples de cette puissance salomonienne sur les esprits rebelles. L'existence ontologique des djinns et de Salomon affirmée par le Coran donne ainsi au texte qui les mentionne une crédibilité plus grande que s'ils étaient mentionnés dans un récit de fiction. Ou disons encore que ce que nous appelons fiction cesse de l'être si les acteurs sont authentifiés par le Coran. Il en est presque de même de la Ville merveilleuse. Elle acquiert un statut d'authenticité dès l'instant qu'elle est mise en relation avec la toute puissance de Dieu. Dans notre récit, la Ville de Cuivre est attestée par le djinn que rencontre Mûsa. Pour une conscience arabomusulmane de l'époque classique, le merveilleux était facilement intégré dans la réflexion religieuse. Le croyant ou le savant ne voyait pas de contradiction ni une atteinte à la logique des choses en parlant d'animaux, de plantes ou de réalisations humaines appartenant au merveilleux. Tout être, toute chose, tout événement qui pouvait émerveiller l'homme était considéré comme un signe qui pouvait conduire sur le chemin de la reconnaissance de la grandeur et de la puissance divine. C'était d'ailleurs-là, une des fonctions essentielles du merveilleux comme l'avait compris les grands compilateurs des $a j \hat{a}^{\prime} i b$ et gharâ'ib dont les plus illustres représentants demeurent Qazwîni et Dimashqi.

La littérature arabe, notamment celle des géographes et des cosmographes a été très tôt marqué et influencé par les récits porteurs de merveilleux. Dès le IXe siècle circule l'anonyme suivant : akhbâr al-Sîn wa l-Hind (relation de la Chine et de l'Inde) et au Xe s on connaît ajâ'ib al-Hind (les Merveilles de l'Inde). Les Prairies d'or (murûj al-dhahab) de Mas ${ }^{\circ}$ ûdi reste encore fidèles à ce genre comme après lui Abu Hamid al-Gharnâti, Qazwîni et l'auteur de l'Abrégé des Merveilles que nous avons déjà cités plus haut ou un Ibn al-Wardi (XVe s.) avec la Perle des Merveilles. L'agiographie et l'histoire n'échappe pas non plus à cette règle si l'on considère les nombreuses histoires des prophètes (qisas al-anbiyấ) dont al-Tha ${ }^{\circ}$ âlibi, par exemple, représente la forme achevée. Tabari (Xe s.) dans sa monumentale histoire est loin de rejeter des récits que nous 
classerions aujourd'hui volontiers parmi les légendes et les contes. Et Jâhiz (776-868) de rappeler déjà dans le kitâb al-hayawân (le livre des animaux) que si Dieu avait créé le monde et avait parsemé dans sa création des phénomènes étranges, c'était pour que l'homme doué de raison décripte ces signes et y reconnaisse la grandeur de Dieu, car la plus minuscule créature est encore un signe de Dieu ${ }^{1}$. Qazwîni reprendra une argumentation semblable dans son ajấib al-makhlûqât.

Le thème du merveilleux a été traité au colloque de 1974, où M. Troupeau insiste sur ce lien : " A propos des merveilles de la création... il est intéressant de constater que jamais, dans le Coran, ces merveilles ne sont désignés, ne sont nommées, par un dérivé de la racine ${ }^{\circ}$ ajiba; elles sont toujours désignées par le mot âya signe. Cinq fois dans le Coran on trouve un long verset qui énumère toutes ces merveilles et dans les cinq versets nous avons cette finales stéréotypée : en cela sont des signes pour un peuple qui raisonne (ya ${ }^{\circ}$ qilûna), qui croit (yu'minûn), qui réfléchit (yatafakkarûn), ou pour ceux qui sont doué d'esprit (ûli l-albâb), c'est -à-dire simplement qui ont, qui possèdent non pas le ${ }^{\circ}$ aql mais le lubb, ce vieux mot sémitique qui signifie coeur à l'origine, comme en éthiopien je crois, et ensuite raison, esprit" 2 .

Pour le croyant musulman de l'époque classique la raison et la foi trouvaient dans le merveilleux non pas l'occasion du doute, comme le ferait un esprit critique du XXe s. occidental, mais la chance offerte à l'homme pour qu'il développe son intelligence et pénètre un peu plus le secret de la création dans laquelle il ne saurait y avoir de ruptures. La création est essentiellement une, et les êtres qui sortent de l'ordinaire pour un Occidental sont au contraire l'expression de la volonté de Dieu pour un Musulman.

Cette introduction doit nous faire comprendre que le récit de la Ville de Cuivre, en raison de son lien avec le cycle de Salomon n'appartient donc plus unilatéralement à la fiction, mais aux signes multiples qui guident le croyant vers l'Unicité de Dieu, cette attestation que les pêcheurs noirs à la fin du récit tiennent à rappeler. En effet, la fin du conte est un hymne à l'Unicité divine. Les Noirs,

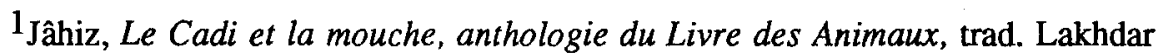
Souami, Paris, Sindbad, 1988, pp. 53-58.

${ }^{2}$ L'Etrange et le merveilleux dans l'Islam médiéval, Paris, ed. J. A., 1974, p. 42. 
pêcheurs des vases de Salomon ont appris la shahâda, et rien d'autre, de la bouche d'un personnage qui surgissait parfois de l'eau de la mer et qui se faisait appeler Abu I-Abbâs al-Khidr. Ne fallait-il pas d'une certaine manière qu'il en soit ainsi, que les vases de Salomon soient en effet repêchés par des croyants et non des infidèles ? Que des peuples, coupés de toute civilisation musulmane, aient eu connaissance de l'islam sans l'intermédiaire de Musulmans est encore un fait merveilleux, au diapason de tout du reste du conte. Outre cette signification, le conte s'empare aussi de données historiques et véhicule ainsi un implicite dont il faut rendre compte.

\section{La structure du conte}

Si l'on remarque quelques contradictions dans la chronologie des personnages, le récit lui-même ne manque pas d'incertitude. Au début du conte il est question d'un homme qui aurait découvert les vases de Salomon après avoir été poussé vers l'Inde (inhadarû ila bilâd al-Hind $)^{1}$ (p. 203). Sur le rivage où la troupe accoste, vivent des Noirs qui ne parlent pas arabe sauf leur roi, et qui ne connaissent surtout rien de l'islam (p. 204). Car, comme explique leur roi, personne ne s'était encore rendu chez eux. Or la recherche de ces vases se fera non en allant vers l'Inde mais vers l'Occident maghrébin et l'al-Andalus. Et les Noirs que rencontrera Mûsa, seront en quelque sorte musulmans, puisqu'il connaissent l'Unicité de Dieu, mais non la prophétie de Mahomet (p. 228).

Le texte des Mille et Une Nuits n'établit pas non plus un lien très étroit entre l'épisode du djinn attaché au tronc d'un vieux palmier et la découverte de la Ville de Cuivre sinon que Mûsa lui demande de leur en indiquer le chemin (pp. 214-215). Or, en toute logique personne ne cherchait la ville mais l'endroit où se trouvaient les vases de Salomon. Le lien entre ces deux épisodes est donc faible. En revanche, l'épisode du djinn attaché au palmier trouve sa justification

${ }^{1}$ Rappelons que $\operatorname{Mas}^{\circ}$ ûdi atteste une ville de cuivre située sur la côte de l'océan indien à l'extrémité du désert de l'Inde, vol. II, p. 547. 
dans le conte en ce sens qu'il illustre la force de Salomon. C'est lui en effet qui a puni ainsi le djinn parce qu'il s'opposait au mariage de Salomon et de la fille du roi de la mer dont il était le lieutenant. Salomon exigeait aussi de briser l'idole que vénérait ce djinn et où il avait établi sa demeure (p. 211). Le conte évoque encore là un thème inattendu : celui des mariages mixtes entre djinns et humains ${ }^{1}$ dont la tradition arabo-musulmane semble avoir été friante un temps.

La même artificialité se retrouve entre l'épisode de la découverte du château renfermant le tombeau de Shaddâd ${ }^{2}$ (pp. 208-210) et celle d'un cavalier en cuivre (fâris min nuhâs) dressé sur un piédestal ( $\mathrm{p}$. 210). Ici il y a purement juxtaposition de deux découvertes faites au hasard du chemin.

Si l'on considère à présent l'ensemble du conte, on observe que le cadre général est celui de la recherche des vases de Salomon. Dans la situation initiale, Abd al-Malik exprime le désir de les posséder. Le récit commence donc bel et bien sur le "manque" des vases. La situation finale comble ce manque, puisque l'expédition réussit à ramener les vases à Damas auprès du calife. La transformation est à proprement parler le voyage entrepris par trois personnages : Tâlib Ibn Sahl le voyageur de Abd al-Malik, Abd al-Samad le voyageur de Abd al-Azîz le frère du calife et enfin Mûsa Ibn Nusayr, le conquérant du Maghreb. On ne peut pas parler d'épreuves véritables en dehors de la mention que le voyage était en soi périlleux et difficile en raison des déserts à traverser et des distances à franchir. Les quatres rencontres : le château de Shaddâd, le cavalier en cuivre, le djinn emprisonné et la Ville de Cuivre sont des épisodes qui suscitent chacun le merveilleux mais qui demeurent disparates dans leur juxtaposition.

Nous pouvons donc conclure à propose de la structure de ce conte qu'il est composé de deux récits indépendants : la recherche des vases de Salomon et la découverte fortuite de la Ville de cuivre

${ }^{1}$ Les Mille et Une Nuits font état de ce genre de mariages, par exemple celui de Hasan al-Basri avec une djiniyya : Manâr al-Sana ; celui de Sayf al-Mulûk avec la djinniyya Badî $^{\circ}$ at al-Jamâl.

${ }^{2}$ Le personnage de Shaddâd nous renvoie directement à une autre ville célèbre Iram aux Colonnes que Tabari situe dans le Hadramawt (Tabari, De la création à David, pp. 56-63). 
auxquels ont été ajoutés des épisodes qui relèvent du même merveilleux comme l'apparition également fortuite du château de Shaddâd, le cavalier en cuivre et le djinn rebelle attaché à un palmier. Mais voyons à présent les caractéristiques de cette Ville de Cuivre.

\section{La Ville de Cuivre}

Relevons d'abord la place qu'occupe le récit de la Ville de Cuivre dans l'ensemble du conte dans l'édition al-maktaba l-thaqâfiyya.
A) Introduction :

Préparatifs et départ de Tâlib : pp. 203-206.

Rencontre avec Abd al-Azîz et départ avec Abd al-Samad auprès de Mûsa Ibn Nusayr : p. 207

B) Voyage :

a) découverte du château de Shaddâd ${ }^{1}:$ pp. 207-210

b) découverte d'un cavalier en cuivre ${ }^{2}$ : p. 210 (moitié)

c) rencontre avec le djinn enchaîné à un palmier : p. 210 à 211 (moitié), suivi du récit du djinn rapportant le désir de Salomon d'épouser la fille d'un djinn : pp. 211-214

d) découverte de la Ville de Cuivre : pp. 215-227

\footnotetext{
${ }^{1}$ Par certains aspects, ce château n'est pas sans rappeler celui du récit de Zuhri, alqasr al-mushayyad situé près du puits magique, al-bi'r al-mu attala, dont la particularité est que l'eau se retire, dès que l'on approche. cf. Zuhri, op. cit. p. 267.

${ }^{2}$ Le cavalier fait penser à la statue en or dressée sur le phare de l'île d'Arîn et évoquée par Zuhri. Cettte statue a la main droite tendue vers l'ouest. Sa main indique un mouvement comme celui du cavalier indique la direction à prendre pour continuer le chemin. cf. Zuhri, op. cit. p. 36.
} 


\section{Conclusion :}

a) recouvrement des vases de Salomon : pp. 227-228

b) retour auprès du calife Abd al-Malik : p. 229

Ce rapide coup d'oeil montre que le récit de la Ville de Cuivre occupe douze pages sur un total de vingt-six. Elle est donc bien la partie la plus importante du conte qui mérite d'être détaillée selon les différents thèmes qu'elle véhicule.

\section{L'apparition de la ville}

Après que la troupe de Mûsa se fût éloigné du djinn, la Ville de Cuivre leur apparut soudain (wa idha bi... ) (p. 215). Sa muraille se présentait comme une totalité sans faille : un bloc de montagne ou une coulée de fer ( $k a^{\prime}$ annahu qit ${ }^{\circ}$ atun min jabalin aw hadîdin subba fi qâlibin ) (p. 215) ${ }^{1}$. L'immensité de cette ville est telle qu'un accompagnateur met deux jours pour en faire le tour à dos de chameau. Lui non plus ne découvre pas de porte pour y entrer. Au bout de trois jours d'attente et de réflexion, Mûsa, Tâlib et Abd alSamad grimpent sur une montagne voisine et aperçoivent ainsi la ville la plus grande qui soit ( la... $a^{\circ}$ zam minha) plantée d'arbres fruitiers, arrosée de cours d'eau abondants, mais vide et sans vie. Seuls des hiboux et des corbeaux planent sur la ville.

Mûsa aperçoit soudain sept écriteaux en grec qu'Abd al-Samad déchiffre (p. 216). Les Mille et Une Nuits donnent d'abord un texte en prose avertissant l'homme de la fragilité de la vie, du temps qui passe et de l'inéluctable destin qui mène à la mort. Puis le récit en prose est doublé par quelques vers qui reprennent le thème précédent. Dans notre édition, le conteur donne le contenu de quatre de ces écriteaux suivis chaque fois de leur doublet en vers (pp. 216-217). Il faut relever ici une imprécision du récit. En effet, ces plaques (alwâh) en marbres qui brillent au loin ( $\left.\min a l-b u^{\circ} d\right)$ sont-elles posées sur la ville ou bien se trouvent-elles dans la montagne? Ce n'est qu'après

${ }_{1}^{1}$ Allusion coraniqueà de la coulée d'une muraille. 
avoir lu ces écritures, que Mûsa et ses amis descendent de la montagne (p. 218) et cherchent un moyen (hîla) pour pénétrer dans la ville pour y voir les choses merveilleuses $\left(a l-{ }^{\circ} a j a ̂ ́ i b\right)$. Le mot hîla est important, non seulement parce qu'il revient fréquemment dans les propos d'Abd al-Samad, mais aussi parce qu'il désigne une ruse qu'il doit mettre en oeuvre. Nous sommes évidemment dans un contexte de magie et de fabrication de talismans qui doivent déjouer la force des sortilèges. En Egypte, cette pratique, propre aux pilleurs de tombes, était bien connue. Ils fabriquaient des amulettes pour se prémunir contre tout châtiment possible des morts dont ils venaient violer le silence. Ce n'est donc pas pour rien qu'Abd al-Samad, vivant en Egypte auprès de Abd al-Azîz, sera le seul à connaître les formules magiques qui le sauveront en haut de la muraille.

\section{La prise de la ville}

Pour grimper sur le mur de la ville, les hommes décident finalement de faire construire un échelle par des menuisiers et des forgerons. Il faudra un mois pour mener à bien cet ouvrage (p. 217). Lorsque le conteur fait revenir la troupe chez les menuisiers et les forgerons, il semble oublier que la ville se situe dans un endroit éloigné de toute vie humaine. Faut-il sous-entendre que Mûsa est revenu jusqu'en terre habitée ? En tous les cas, le récit des Mille et Une Nuits ne semblent pas indiquer cela.

Douze compagnons grimperont sur cette échelle, mais arrivés au sommet de la muraille, l'un après l'autre se met à battre des mains et à crier : tu es beau ! et finalement se jette dans la ville où il se fracasse les os et meurt. Dans la version d'al-maktaba l-thaqâfiyya, il n'est pas réellement question du rire des douze compagnons, sauf pour Abd al-Samad (p. 219) qui réussit à se maintenir au sommet de la muraille grâce à la récitation de la basmalla et de certains versets coraniques. Il voit maintenant la cause de la mort de ses prédécesseurs : dix jeunes filles (jâriya) belles comme des lunes appelaient les compagnons vers elles. Ils avaient également l'impression d'avoir à leur pied une étendue d'eau (bahran mi al$\left.m \hat{a}^{\prime}\right)$. Abd al-Samad ajoute que, sans doute, les anciens habitants de cette ville avaient dressé ces sortilèges (mirage des jeunes filles- 
djinns et du lac) pour se protéger de tous ceux qui avaient l'intention d'y pénétrer. Nous retrouvons ici une opinion commune répandue en Egypte chez les fouilleurs de tombes qui étaient sensées se protèger des violeurs, en provoquant la mort de ceux qui ne savaient leur opposer un pouvoir magique plus grand que le leur. Il est clair que la récitation du Coran était le meilleur moyen pour déjouer la magie (sihr) de la Ville de Cuivre.

Abd al-Samad marche alors sur la muraille et rencontre deux tours en or. Sur la porte de l'une d'elles est gravée une image (sûrat) d'un cavalier de cuivre ${ }^{1}$ ayant la paume de la main ouverte (p. 220). Il suffisait de frotter douze fois le clou qui se trouvait dans son nombril pour que la porte de la tour s'ouvre. Abd al-Samad peut ainsi descendre dans la ville où il rencontre des gens et un vieillard comme momifiés. Le vieillard porte sur lui des clefs avec lesquelles, enfin, il réussit à ouvrir la ville (p. 221).

\section{L'intérieur de la ville}

Les trois pages qui suivent font la description de cette ville. Il est question d'abord des habitants figés sur place, morts, mais donnant l'impression de reprendre la vie à tout instant. La vie dans les quatre souks est simplement arrêtée. Tout est en place pour le négoce et le commerce, l'or, les bijoux, les pierres précieuses, les parfums, l'ivoire, l'ambre, le musc, bref tous les produits de luxe et les produits d'usage courant (p. 221).

Après la traversée des souks, vient une série de monuments. Un château d'abord avec quatre immenses salles d'accueil (majâlis). Dans chacune, coule une fontaine et passe un cours d'eau. Chacune de ces quatre rivières se jette dans un immense lac (buhayra ${ }^{\circ} a z \hat{\imath} m a$ ). Nous voyons Mûsa et sa suite traverser ces quatres salles immenses remplies de richesses inouïes ; or, argent, pierres précieuses, armes, vaisselle fine... A partir de ce château, ils empruntent un couloir

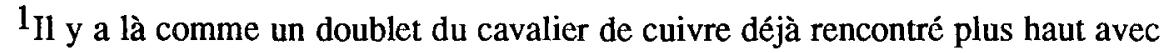
cependant la différence que celui-ci ne se dresse pas sur un socle comme une statue mais était gravé sur la tour en or. 
(dihlîz), dont l'entrée est dissimulée par un rideau de soie, mais qu'Abd al-Samad réussit à ouvrir grâce à son savoir ( $\mathrm{ma}^{\circ}$ rifat et son courage (shaja $\hat{a}^{\circ} a$ ) et son habileté $\left(\operatorname{bar} \hat{a}^{\circ} a\right.$ ). Ce couloir les conduit dans une autre salle $\left(q \hat{a}^{\circ} a\right)$ immense et richement ouvragée, construite en marbre, si poli quelle donnait l'impression d'une eau courante, (p. 223), couverte d'un dôme sous lequel se dressait un dais (khayma mina $l$-dîbaj) soutenu par quatre pilastres d'or rouge, renfermant un lit (p. 224).

\section{La jeune fille}

L'objet le plus fabuleux de cette ville magique était sans contre dit le lit (sarîr) brodé de perles et de pierres précieuse sur lequel reposait une jeune fille admirablement belle, gardée par un esclave blanc et un esclave noir, en armes p. 224). L'esclave noir portait une écriteau sur lequel était gravée la basmallah en prose, suivi de certains noms de Dieu et des considérations sur la fragilité de la vie humaine. En bas de cet écriteau venaient une nouvelle fois des vers, corroborant le sens du texte en prose (p. 225). Cette insistance sur l'unicité et la puissance de Dieu introduit l'idée que la ville fut construite par Salomon. Puis vient de nouveau une considération semblable, en prose (p. 226). Ces deux pages qui font si explicitement référence à l'islam et à l'éthique musulmane apparaissent comme un ajout évident, un développement dans lequel le conteur peut librement exposer ses vues sur des problèmes aussi difficiles que le temps et la mort ${ }^{1}$. Le conteur s'interroge sur tous ceux qui jadis étaient de grands hommes : les rois de Chine, Shaddâd, Nemrôd, Pharaon... les représentants traditionnels des tyrans qui,après avoir soumis les peuples, ne purent échapper à la mort.

Tâlib Ibn Sahl veut ramener la jeune fille et la donner au calife de Damas, mais au moment où il la touche, il est tué sur place par les deux gardes : l'un lui tranche la tête et l'autre le transperce de sa lance (p. 227). Sur ce, Mûsa donne l'ordre de fermer la porte de la ville comme elle était auparavant (an yaghliqû l-bâb kama kâna) et quitte la

${ }_{1}$ Mardrus par exemple omet toutes ces considérations dans sa traduction 
ville avec sa troupe. Nous pouvons encore trouver là, une autre manière d'agir des violeurs de tombes égyptiennes, qui après leurs infractions, rebouchaient les tombes comme elles étaient. Ainsi, leur méfait demeurait-il inconnu et impuni.

La troupe arrive bientôt sur le bord de la mer, au pied d'une grande montagne et rencontre les Noirs qui connaissent l'endroit où gisent les vases de Salomon. Mais déjà la Ville de Cuivre est oubliée et le conteur islamise les Noirs (p. 228), en recourant à un autre type de merveilleux : l'apparition d'Abu l-Abbâs al-Khidr ${ }^{1}$. Cette figure coranique pose une nouvelle fois la question de l'historicité de ce récit. La Ville de Cuivre est certes une légende, mais une légende appuyée sur des noms connus, comme nous le voyons à présent.

\section{L'histoire explicite}

Dans le conte de la Ville de Cuivre certains éléments sont facilement datables dans une histoire événementielle. D'autres demeurent au plan d'hypothèses.

Les données qui font directement allusion à l'histoire arabe portent d'abord sur Abd al-Malik Ibn Marwân (m. 705) qui est calife à Damas, le cinquième successeur de $\mathrm{Mu}^{\circ}$ awiyya, régnant de 685 à sa mort en 705. Il est le fondateur de la branche marwanide des Umayyades. Ce fut un calife qui dut lutter contre l'éclatement de l'empire. En 692, il réussit à reprendre La Mekke où règnait l'anticalife Ibn al-Zubayr. Grâce au fameux gouverneur de l'Irak, al-Hajjâj il réprima des récoltes chiites et kharedjites en Syrie et en Irak. Vers la fin de sa vie il soumit la Kâhina au Maghreb et ouvrit définitivement l'Ifriqiya aux Arabes. C'est lui, qui, dans le conte,

${ }^{1}$ Ce personnage semble bien être une fabrication complexe à partir d'un Abu 1Abbâs, célèbre traditionniste et Khidr, le compagnon mystérieux de Moöse. Ce personnage a donné lieu à bien des spéculations pour savoir à quel prophète d'Israël il pouvait bien correspondre : Elie, Ezéchiel... ? Son nom est tout à fait à l'image du conte entier qui réunit différentes traditions appartenant au fond judéochrétien, musulman mais aussi grec et égyptien. 
désire voir ces fameux vases dans lesquels Salomon avait jadis enfermé les djinns rebelles.

Il est également question de son frère Abd al-Azîz qui à l'époque gouverne l'Egypte et caressait l'espoir de lui succéder. Il meurt un an avant Abd al-Malik supprimant ainsi tout danger pour son frère.

Mûsa Ibn Nusayr est le grand conquérant de l'Afrique du Nord et de l'Espagne avec son lieutenant Târiq qui pénètre en al-Andalus en 711.Il gouverne l'Ifriqiya à partir de 708. En 713, les troupes de Mûsa prennent Narbonne, alors capitale wisigothique. Le conte, en revanche, commet une première entorse à l'histoire avec ce personnage. En effet, Mûsa n'est véritablement maître de l'Afrique du Nord qu'en 708, c'est-à-dire trois ans après la mort d'Abd alMalik. Dans les Mille et Une Nuits, Mûsa, une fois revenu à Damas, demande au calife la permission de se retirer à Jérusalem pour y finir ses jours dans la prière. Cette fin pieuse est encore en contradiction avec l'histoire véritable, car Mûsa revient à Damas en 715 en compagnie de Târiq Ibn Ziyâd ramenant un riche butin. Mais les deux hommes tombent rapidement en disgrâce sous le successeur d'Abd al-Malik. Mûsa est même accusé d'avoir détourné une part des biens. Il mourra en exil et pauvre.

La chronologie des personnages est encore plus rudement mise à l'épreuve avec la mention du poète al-Nâbigha al-Dhubiyâni 1 comme assistant à la réunion où le calife formule le voeu de voir les vases de Salomon. Le poète cite deux vers : "A propos encore de Salomon : Dieu lui dit : prends le califat et gouverne comme un sage! Celui qui t'obéira, récompense-le. Celui qui désobéira, emprisonnele pour l'éternité". Or le poète cité appartient à l'anté-islam. Il aurait vécu à la fin du VIe $s$. ou tout au début du VIIe siècle, soit un siècle au moins avant le calife ! Mais pourquoi sa présence ? Et surtout pourquoi ces deux vers ? C'est que Nâbigha al-Dhubiyâni est célèbre pour deux vers dans lesquels il rappelle que Dieu a permis la construction de Tadmor (Palmyre en Syrie) à des djinns et a donné à Salomon le pouvoir de les y enfermer ${ }^{2}$. C'est cette deuxième

1 Al-maktaba 1-thaqâfiyya, p. 205.

2 Al-Nâbigha al-Dhubiyâni, divan, éd. Ahlwardt, 5 , vers 22 et 23. 
composante qui rejoint le thème du conte. Le conte fait allusion également à des données historiques implicites.

\section{Les données implicites}

Le conte nous renvoie également à un contenu implicite plus difficile à déterminer que celui de l'histoires des personnages. En effet, pourquoi des villes mystérieuses, des châteaux mystérieux font-ils tant allusion à la mort ? Un fait est sûr. C'est sous les Umayyades que l'Egypte est explorée plus que jamais. Or l'Egypte livre aux chercheurs et aux voleurs de tombes pharaoniques, de nombreuses villes abandonnées et d'importantes nécropoles. L'imagination populaire n'avait aucun mal à s'emparer des découvertes qui pouvaient être faites surtout s'il s'agissait de tombes de Pharaons. Leurs fabuleuses richesses, leurs tombeaux richement ornés et peints pouvaient donner l'impression que la vie allait reprendre. Les écritures indéchiffrables devenaient aussitôt mystérieuses, comme la mort, que seul le sage et le savant pouvaient interpréter. Il est donc facilement compréhensible que certaines versions situent directement la Ville de Cuivre en Egypte et que le château de Shaddâd renferme autant de tombes. C'est en fait une nécropole. L'inviolabilité de la ville par Mûsa peut renvoyer à l'inviolabilité des tombes pharaoniques. Un châtiment terrible menaçait toujours les violeurs. La rumeur populaire savait qu'il ne fallait pas déranger les morts sous peine d'être inquiété. Mûsa est lui aussi fasciné par cette ville comme pouvaient l'être les Egyptiens découvrant une ville abandonnée ou des tombeaux anciens. Les Mille et Une Nuits en font en quelque sorte un violeur de sépulture.

Que dire des grottes qu'habitent les Noirs non loin de la mer à flanc de montagne? La région la plus connue pour ses habitation troglodytes se trouve dans le pays berbère du sud Tunisien. Le conte mentionnant le conquérant de l'Afrique du Nord pourrait faire allusion à cette région. Mais cette hypothèse complique alors l'explication des pêcheurs noirs. Les Berbères ont la peau blanche et sont difficilement assimilables aux Noirs. Le récit redevient cohérent si l'on place la Ville de Cuivre dans le Haut Nil. En fait, il se peut que deux traditions différentes, ou plus, se superposent ici. 


\section{La ville et la mère}

Depuis la plus haute antiquité, certains textes établissent explicitement un lien entre la ville et le corps de la femme. Ainsi, dans la tradition chrétienne primitive, trouvons-nous le thème de la Jérusalem céleste et de Babylone comparées à une mère. Le premier apparaît dans l'explication donnée par l'épître aux Galates, des deux alliances avec Agar et Sara, la servante et la femme d'Abraham, nous trouvons une première analogie entre l'alliance représentée par Sara (donnant naissance à Isaac l'ancêtre d'Israël) et Jérusalem. Or cette alliance acquiert une nouvelle dimension dans la métaphore suivante : "Mais la Jérusalem d'en haut est livre et elle est notre mère... (Gal. 4, 26). A la Jérusalem céleste s'oppose dans l'Apocalypse, Babylone la Grande, pseudonyme de Rome. Or voici comment l'Apocalypse décrit cette ville. "Alors l'un des sept Anges aux sept coupes s'en vint me dire: Viens, que je te montre le jugement de la Prostituée fameuse, assise au bord des grandes eaux ; c'est avec elle qu'ont forniqué les rois de la terre, et les habitants de la terre se sont saoulés de vin de sa prostitution. Il me transporta donc au désert, en esprit. Et je vis une femme, assise sur une Bête écarlate couvertes de titres blasphématoires et portant sept têtes et dix cornes. La femme revêtue de pourpre et d'écarlate, étincelait d'or, de pierrerie et de perles; elle tenait à la main une coupe en or, remplies des répugnantes impuretés de sa prostitution. Sur son front, un nom était inscrit - un mystère! Babylone la Grande, la mère des répugnantes prostituées de la terre... Et cette femme-là, c'est la Grande Cité, celle qui règne sur les rois de la terre". (Apocalypse, 17, 1-18).

La tradition chrétienne n'innove pas à proprement parler. Elle se situe en continuité de la tradition juive qui connaissait déjà depuis très longtemps le thème de Jérusalem, fille de Sion, comparée à une Vierge. Dans un passage fameux, le prophète Osée compare Israël tout entier à une femme infidèle : "Accusez votre mère, accusez-la! Car elle n'est plus ma femme et je ne suis plus son mari. Qu'elle bannisse de sa face ses prostituions, d'entre ses seins ses adultères ; 
sinon, je la déshabillerai toute nue et la mettrai comme au jour de sa naissance..." (Osée, 2, 4-6).

Quant au conte des Mille et Une Nuits, pouvons-nous établir cette relation entre la ville et la mère ${ }^{1}$ ?

Les images les plus pertinentes qui rappellent la mère sont avant tout le palais que renferme la ville et qui est lui-même composé de quatre grandes pièces dont l'une donne sur un couloir (dihlîz) qui mène à une nouvelle salle $\left(q \hat{a}^{\circ} a\right)$ surmontée d'une coupole et renfermant un dais en velours, à l'intérieur duquel est dressé un lit sur lequel est couché une jeune fille qui semble dormir. Le mouvement général esquissé dans cette description est celui de la descente et de l'enchâssement. Abd al-Samad, du haut de la muraille, descend dans la ville après avoir franchir une porte et traversé un couloir ; Mûsa, une fois à l'intérieur de la ville, passe de couloirs en salles et dans chaque salle, il découvre des coffres et des armoires remplis de richesses inestimables.

Il y a là un emboîtement évident : ville---souk---château---salle--coffre/armoire---richesse---objet du désir.

Cet emboîtement nous renvoie directement au complexe de Jonas ou de la gullivérisation, euphémisation de l'avalage comme l'a montré Durand ${ }^{2}$. Or, explique-t-il : "La gullivérisation s'intègre donc dans des archétypes de l'inversion, sous-tendue qu'elle est par les schèmes sexuel ou digestif de l'avalage, surdéterminée par les symbolismes du redoublement, de l'emboitement. Elle est inversion de la puissance virile, elle confirme le thème psychanalytique de la régression du sexuel au bucal et au digestif" 3 . Il nous faudra déterminer plus loin de quelle régression il s'agit dans notre conte. Relevons encore que l'isotopie du féminin est soulignée dans la Ville de Cuivre par la présence des dix djinniyyât qui fascinent et attirent irrésistiblement les compagnons qui grimpent sur la muraille, sauf Abd al-Samad qui oppose à leur charme la puissance désenvoûtante

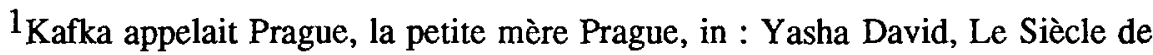
Kafka, Paris, Centre G. Pompidoun 1984, p. 89.

2Durand, Gilbert, Les Stuctures anthropolmogiqueq de l'imaginaire, Paris, Dunod, 1984, p. 233.

3 Durand, op. cit. p. 243. 
des versets coraniques. Leur beauté de lune n'est rien à côté de la beauté et de l'efficacité du Coran. Aussi sont-elles vaincues sans peine par la basmallah d'Abd al-Samad et elle ne réussissent donc pas à l'attirer dans le mirage de la mer qui paraît s'étendre au pied de la muraille. De part la présence des djinniyyât, la ville devient la grande avaleuse d'hommes. Elle attire en son sein, les hommes qui ont réussi à grimper sur la muraille, puis elle les broye dans un fracas terrifiant comme une bête sauvage le fait de la proie qu'elle avale. De ce point de vue, la ville fonctionne comme la mer, la grande avaleuse par excellence, symbolisée dans le mythe de Jonas. Dans ce récit, la mer et le monstre marin ne font qu'un et l'homme est englouti vivant par le monstre. Dans un grand nombre de contes des Mille et Une Nuits, le héros est également victime de la mer, mais en est rejeté afin qu'il puisse parachever sa quête initiatique ${ }^{1}$. Ce thème de l'avalage est donc singulièrement souligné dans ce conte. Sa force métaphorique nous renvoie à cet autre avalage fantasmatique qu'est la relation sexuelle évoquée dans le rapprochement de l'isotopie masculine.

Cette première approche de la ville en tant que signifiant du féminin et plus précisément de la mère doit en effet être mise en relation avec l'isotopie du masculin qui la parachève et donne le sens complet au conte. Pour Freud, "montagne et rocher sont des symboles du membre masculin... et la clef qui ouvre est incontestablement un symbole masculin" 2 . Mais ce qui nous intéresse ici, c'est que ces symboles entrent eux-mêmes dans une nouvelle signification qui est le rapport sexuel directement. A ce propos, Freud écrit encore : "On peut citer, comme se rapportant a cette catégorie,des activités rythmiques telles que la danse, l'équitation, l'ascension... Ajoutons encore certaines activités manuelles et, naturellement, la menace avec une arme"3. Il est particulièrement mis en évidence dans le conte que Mûsa grimpe sur une montagne pour mieux voir la ville d'en haut, puis il fait escalader

${ }^{1}$ Cest particulièrement frappant dans Qamar al-Zamân et Budûr, Sayf al-Mulûk et Badî̊at al-Jamâl, Jullanâr et Badr Bâsim...

${ }^{2}$ Freud, Introduction, p. 143.

${ }^{3}$ Freud, Introduction..., p. 142. 
par Abd al-Samad, la muraille, sans porte (sans sexe), grâce à une échelle qui, selon al-Gharnâti, est posée sur des blocs de rochers. Il se fait également ouvrir avec une clef, la tour qui, du sommet de la muraille, mène dans la ville, puis la porte de la ville, enfin le couloir menant à la jeune fille (p. 220). A côté de celle-ci, se tiennent deux gardes armés qui transperceront Tâlib au moment où il la touche. Le conte se termine donc sur la prohibition totale de la relation sexuelle voire du viol. Il nous faut à présent préciser la nature réelle de la femme dont la ville ou la jeune fille est la métaphore.

\section{Le corps de la mère comme objet de désir}

Cette première métaphorisation ou mieux encore cette allégorie peut être davantage explicitée si l'on se reporte à l'analyse psychanalytique et particulièrement lacanienne. De quoi s'agit-il ? Ou plutôt, comment comprendre les différents essais des compagnons de Mûsa pour grimper sur la muraille, essais soldés régulièrement par un échec. On pourrait articuler ces épisodes comme l'affrontement de l'ordre de la loi et de l'ordre du symbolique, auxquels Lacan ajoute l'ordre de l'imaginaire.

Le Calife de Damas représente cet ordre, en ce sens qu'il décide d'envoyer quelqu'un à la recherche des vases de Salomon dès qu'il en entend parler. L'immédiateté de ce désir de prendre possession des vases est symptomatique. Le Calife ne sait rien de ce qui va arriver à Mûsa, à savoir l'apparition inattendue de la ville de cuivre. Il lui ordonne néanmoins d'aller chercher les vases de Salomon. C'est eux l'objet de son désir. Tout se passe comme s'il ne pouvait dominer ce désir déclenché par le récit d'un voyageur ou d'un conteur, c'est-à-dire par l'ordre symbolique. La parole, on le sait, est en effet concomitante au désir. Dès que l'homme apprend à parler, il apprend à désirer ${ }^{1}$. Mais le désir ne peut aller sans rencontrer la loi.

1 Pommier dira que "l'entrée dans le langage lui-même" est en quelque sorte "l'entrée dans la jouissance phallique... En même temps, le fait de parler apporte les repères symboliques du complexes d'Oedipe et introduit le type de jouissance qui est propre à ce complexe" (Le Dénouement d'une analyse, p. 122). 
Le conte illustre l'ordre de la loi, en décrivant avec abondance les différents tableaux qui jalonnent le parcours de Mûsa et sur lesquels il est rappelé à l'homme l'inéluctable destin de la mort. A la jouissance ${ }^{1}$ que veut atteindre le Calife, en s'emparant de l'objet de son désir, s'oppose la loi de la mort. Or sur quoi insistent ces pancartes, sinon sur le renoncement, sur la prise de conscience que l'homme ne fait que passer, que tous les biens de la terre sont éphémères. Il y a là une morale évidente recommandant la prise de distance par rapport à tout ce qui fantasmatiquement devrait faire jouir. Mûsa, en recopiant les textes que lui lit son compagnon, intègre la loi, c'est-à-dire accepte d'une certaine manière de renoncer. Si Mûsa est sauvé, c'est qu'il accepte la loi qui donne une limite à la jouissance infinie. La résolution du complexe d'Oedipe est une humanisation du désir par l'acceptation de la loi du père. Les textes antérieurs aux Mille et Une Nuits montre très bien ce renoncement, puisqu'il ne réussit pas à pénétrer dans la ville. En revanche, la non intégration de la loi est mise en images par les compagnons qui, pour de l'argent -l'éternel objet de désir- , cherchent à escalader la muraille de la ville et notamment Tâlib Ibn Sahl qui veut s'emparer de la fille (p. 227). Ainsi, le récit de la Ville de Cuivre situe la jouissance en fonction de deux axes. Dans le premier cas, elle est non corrélée à la loi et conduit à la folie et la mort des compagnons qui ont escaladé la muraille. Dans le second cas, elle est soumise à la loi et introduit le désir qui est de renoncer à la jouissance comme le fait Mûsa en ne touchant pas à la fille. L'acceptation de la loi, la reconnaissance du père fait sortir du désir de la mère, fait accepter en somme la castration. Mais revenons pour l'heure aux compagnons de Mûsa.

La jouissance est tellement forte chez eux, qu'ils sortent même de l'ordre du symbolique. Car ils ne répondent plus à leur compagnons restés au pied de la muraille. Leur parole n'est plus que cris effrayants. La jouissance dans laquelle ils entrent, en voyant les djiniyyât, les conduit irréversiblement à la mort. Car toute jouissance sans frein, désarimée à la loi, sans interdit est une jouissance folle, non corrélée au symbolique. C'est cela le prix de la jouissance, la mort. Le désir qui n'entend pas l'ordre de la loi conduit

1ll faut entendre le mot jouissance dans le sens que lui donne Lacan dans $L e$ Séminaire XX, encore, Paris, Seuil, 1975. 
immanquablement à la mort. Ceci est encore symbolisé par la décapitation de Tâlib Ibn Sahl voulant toucher la jeune fille étendue sur le lit malgré la pancarte qu'elle portait sur elle et qui prohibait toute approche.

En effet, la métaphore la plus forte demeure la jeune fille que Mûsa trouve sur un lit. Au coeur même de la ville, d'emboîtement en emboîtement, Mûsa découvre le sens même de la ville, résumé et concentré dans cette jeune fille, gisante comme si elle était vivante et morte à la fois. La ville toute entière est résumée dans cette métaphore de l'ambiguïté par excellence. La ville morte est le corps de la fille morte. La ville est fascinante comme la beauté de la jeune fille, comme son corps passif dont il ne faut cependant pas approcher. Mais point n'est besoin que Mûsa aille plus loin, le viol est déjà commis, puisqu'il a pénétré dans la ville. Il ne lui restera plus qu'a ramasser les richesses de la ville avant son départ pour que le viol et le vol ne fassent plus qu'un.

La fille ne fait que lui révéler la vraie nature de ce qu'il a pénétré. Non point le corps de la ville, mais le corps de la femme qui tour à tour est l'amante et la Mère. Nous entendons par Mère ce qu'en dit Calligaris. "Ce que j'appelle La Mère, d'ailleurs, n'est pas loin de ce que Lacan appelle La Femme. Car, si La Mère est un mirage pour les deux sexes, c'est qu'elle e fait pas pendant à la défaillance toujours phallique du père : la façon dont sa figure appelle l'être parlant à se perdre en elle n'est pas celle du vide qui fait horreur, voire d'un manque en dernier ressort aussi phallique. La Mère échappe - ce qui se vérifie quand elle est l'Autre effectif du psychotique - au signifiant de la sexuation et présentifie, à cet égard, pour tous, l'Autre sexe, celui que conjuguerait un autre signifiant que le Phalllus. C'est pourquoi aussi sa complétude est terrifiante, parce que rien n'assure l'être parlant que sa propre impuissance phallique suffise à l'y soustraire. La Mere n'existe pas, certes, mais est pour chacun l'horizon de la jouissance, qu'il s'en garde en le regrettant, ou qu'il s'y abandonnne.

J'ai choisi d'appeler La Mère le mirage de la jouissance de l'Autre, parce que, pour le névrosé, ce mirage est en effet maternel. Si c'est l'interdit de l'inceste avec la mère qui sanctionne le ratage du rapport 
parental, rien d'étonnant alors à ce que l'unité de mesure d'un tel ratage soit La Mère, figure de l'inceste"1.

A cet égard, la ville représente bien La Mère comme espace fantasmatique de jouissance, l'espace qui appelle le sujet à s'y perdre comme Oedipe s'est perdu dans sa mère. Ainsi sommes-nous ramenés au complexe d'Oedipe et la relation triangulaire explicitée par Freud et symboliquement rappelée dans le conte par le chiffre trois des trois compagnons qui essaient de grimper sur la muraille sans pouvoir s'y maintenir. Si les Mille et Une Nuits ne mentionnent plus ces trois essais, mais en évoquent douze (un multiple de trois), elles imaginent en revanche le succès d'Abd al-Samad en opposant à la magie de la ville et plus précisément aux dix djinniyyât à l'intérieur de la ville (p. 218), la force des versets coraniques (p. 219), c'est-à-dire en opposant à la fascination du désir le renoncement à la jouissance qu'incarne parfaitement Mûsa mais à laquelle succombe encore Tâlib Ibn Sahl. Et d'Abd al-Samad, il n'est plus question dans le récit de la ville dès l'instant qu'il a ouvert la ville ( p. 219). Certes, il réapparaît dans la suite du conte à propos des vases de Salomon, mais le récit de la ville l'oublie. Il semble bien qu'il faille voir là plus qu'un simple oubli, plus qu'une simple fonction accomplie (celle d'ouvrire la ville). La disparition d'Abd al-Samad est encore une mort symbolique. Lui aussi sur la muraille a battu des mains et a ri comme ses douze devanciers (p. 219). Il a donc lui aussi succombé à la jouissance. Et son engagement dans un long couloir (dihlîz) rappelle trop l'errance dans le corps de La Mère, la prohibition de l'inceste et la mort. Il ne peut en être autrement car "par-dela l'imagerie incestueuse..., La Mère est une unité où l'être parlant, en croyant compléter l'Autre, s'anéantit en se complétant lui-même" ${ }^{2}$. La mort de Tâlib Ibn Sahl, celle des douze compagnons et la disparition (momentanée) d'Abd al-Samad nous renvoient au thème du désir.

${ }^{1}$ Calligaris, Contardo, Hypothèse sur le fantasme, Paris, Le Seuil, 1983, p. 132.

${ }^{2}$ Calligaris, op. cit. p.135. 


\section{Le désir et la loi}

Nous devons remarquer sans peine que le récit commence par un désir. Désir de savoir ce que renferme la Ville de Cuivre, savoir ce qu'elle est. La Ville de Cuivre devient donc ainsi l'objet d'un désir primordial, voire l'hallucination du désir ${ }^{1}$. Et cet objet ou cette ville se situe par rapport à la capitale umayyade sur les limites du monde musulman. Le Maghreb al-Aqsâ au quel est assimilé l'al-Andalus est en effet la terre la plus lointaine où se couche le soleil ; et la ville semble même se situer au-delà de cette frontière du monde de l'Islam. On peut donc dire que que cette ville représente également un objet disparu. Aucun homme en effet depuis Salomon et Alexandre n'a pu l'atteindre. Entre le sujet désirant et l'objet désiré, il y a une distance quasiment infranchissable, un chemin emprunté par personne. Y aller revient dès lors à commencer une quête. Et seule dans cette difficile quête, la ville peut être re-trouvée. Cette distance évoque aussi une formidable séparation, une béance autour de laquelle désormais le sujet a établi sa quête.

Or en psychanalyse, la perte de l'objet n'est pas sans évoquer la mère perdue, sinon la mère morte. La ville est blanche, belle et formidablement riche, métaphore du sein primordial auquel est réduite la mère au stade bucal, sein contenant toute la richesse souhaitable pour une petit être : le lait nourricier. La Ville de Cuivre renfermant un luxe indescriptible d'or, d'argent, de pierres précieuses, de perles, et d'étoffes finement brocardées n'évoque somme toute que cette richesse fantasmatique qui assure le bonheur. Or le lait de la mère, aliment primordial, assure cette fonction du bonheur paradisiaque. Mais tout être humain, un jour doit perdre le sein de sa mère et cette perte est vécue fantasmatiquement comme la perte de la mère totale ${ }^{2}$. Devenir adulte consiste dès lors à guérir de

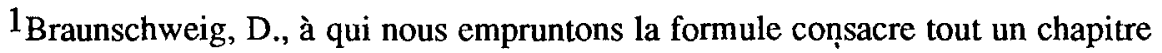
au rêve et à la castration dans : La Nuit, le jour, Paris, Puf, 1975, pp.190-302.

${ }^{2}$ Calligaris écrit encore à ce propos : "Et il faut bien insister sur ceci, que le corps auquel on s'offre comme sein ne s'imagine pas, ne peut s'imaginer, car s'offrir comme sein c'est déjà être perdu en ce corps, au-delà de toute distinction possible" (p. 134, op. cit.). 
cette perte, ou du moins à ne plus en mourir. La quête de l'objet est une première défense contre la dépression que provoque la perte de l'objet et la régression ${ }^{1}$ à laquelle s'est livré le sujet. Abd al-Malik, en entendit le récit de la Ville de Cuivre, c'est-à-dire en prenant conscience de la perte de l'objet, veut calmer en lui la douleur que porte en elle cette déchirure. Aussi veut-il s'assurer que cette ville existe bien, qu'elle est encore là, qu'il pourrait éventuellement s'y rendre ou du moins jouir des richesses qu'on lui en ramènerait. L'angoisse sera moins forte si le témoignage occulaire confirme le récit qui en est fait. Le texte arabe emploie le verbe ${ }^{\circ}$ ayana qui indique bien que Mûsa est chargé de voir cette ville de ses propres yeux.

Peu importe donc qu'Abd al-Malik s'y rende lui-même dans un premier temps. Le plus important est qu'il ait déclenche un processus propre à la quête et qu'il demeure, lui, l'instigateur de cette quête. C'est en fait sa quête. Cette ville est ronde, coulée et moulée en un seul bloc, sans faille. Les vingt-cinq portes n'entament en rien le poli de cette imprenable muraille. Cette enceinte est lisse et impénétrable comme le sein de la mère ${ }^{2}$.

\section{L'étrange inquiétude 3}

Comme la ville, le sein est évanescent et invisible à certaines heures pour réapparaître idéal et étrange à la fois. Son absence provoque la douleur et la peur, sa présence rassure et comble. Ce sein étrange de la mère n'est pas sans ambiguïté. Il devient un lieu angoissant dès l'instant où il n'est jamais définitivement acquis, dès l'instant où il apparaît et disparaît après avoir laissé derrière lui toute la tonalité de l'agréable et du désagréable. Le sein comme la mère est nourricier et frustrant à la fois.

${ }^{1} \mathrm{~A}$ propos de la régression et de ses symboles : Green, A., Sur la mère phallique, in Revue Frt. de Psychanalyse, 1, pp. 1-37.

${ }^{2}$ Spitz, La cavité primitive, in Revue Fr. de Psychanalyse, 1959, 23, pp. 205234.

${ }^{3}$ Freud a traité cette notion dans Essai de psychnanalyse appliquée, Paris, Gallimard, 1980, p. 175. 
Quand Mûsa pénétra dans la ville, tout était en place pour reprendre vie. Les quatre souks étaient remplis de gens et les denrées ne manquaient pas. La ville vivait figée, la ville était morte mais au seuil du réveil. Il était difficile de trancher entre vie et mort. La Ville de Cuivre signifiait en même temps la mort dans la présence des êtres et l'absence dans la vie possible. La frontière entre les deux mondes était devenue fragile, il suffisait de si peu pour que la jeune fille au teint encore rose et aux yeux si vifs se mettent à parler. Et si elle ne faisait que dormir ? Mûsa trouva la ville fort étrange et cette étrangeté l'inquiétait comme s'inquiète l'enfant découvrant l'étrangeté du sexe de sa mère. Cette différence sexuelle vue pour la première fois révèle non seulement à l'enfant que sa mère n'a pas de pénis comme lui, mais que désormais cette absence inscrit une limite irréductible. Il ne sera donc jamais comme sa mère. Et cette première différence lui promet d'autres irréductibilités. C'est dans cette progressive différence avec sa mère que naît la douleur des premières déchirures jusqu'à la séparation définitive. Ce n'est pas pour rien que Mûsa s'évanouissait à tout instant. Le conte est particulièrement précis sur ce détail. Lorsqu'il se trouve sur la montagne, il découvre sept plaques en marbre blanc. Chacune comporte un texte en prose et un texte en vers. Or Mûsa pleure à chaque lecture du texte (p. 215-217). Quand il découvre l'inscription gravée sur les murs du château de la ville (p. 222), non seulement il pleure, mais s'évanouit aussi. Il pleure encore en lisant l'inscription que porte l'un des gardes de la jeune fille (p. 226).

L'insoutenable, comme une obsession, lui revient à la mémoire. Il est entré dans une ville intouchée, vierge, mais il en parcoure maintenant les secrets dédales, les couloirs et les chambres. Il découvre même le secret des serrures magiques. L'espace intérieur dans lequel il est entré le premier devient une étrange scène ${ }^{1}$. La vierge est là sur un lit, allongée et passive, comme si dans son immobilité, elle attendait la visite interdite.

${ }^{1}$ Devereux note que le vertige est souvent en rapport avec les souvenirs de la scène primitive : Devereux, Femmes et mythes, Paris, Flammarion, 1982, p. 52. 


\section{L'inceste}

Mais c'était déjà de trop, Mûsa avait franchit les portes de la ville, il était descendu dans la ville succombant à ses charmes. Et si cette vierge ne faisait en vérité qu'un avec sa mère ? Ce n'est donc pas d'un viol ordinaire qu'il s'agit mais de l'inceste. Or l'inceste rend aveugle. Est-ce aussi pour cela que Mûsa pleure et s'évanouit ? L'inceste doit être puni de mort. Tâlib Ibn Sahl avait tort de toucher à la fille. La punition ne s'est pas faite attendre. Les deux gardes le tuent aussitôt qu'il la touche. Dans la décapitation, nous pouvons voir une image plus forte encore que le simple décollement d'une tête. Il s'agit de la castration au sens que lui donne Lacan. "La castration veut dire qu'il faut que la jouissance soit refusée, pour qu'elle puisse être atteinte sur l'échelle renversée de la Loi du désir"1. Pommier dira de la castration qu'elle "se présente le plus souvent sous la forme d'une angoisse de mort" 2 . Il faut en effet la mettre en relation avec la peur. Or la pire des peurs et la pire des punitions est d'être castré, de perdre le signe qui fait la différence avec la mère. Un sexe manquant fait horreur. Cette peur saisit Tâlib Ibn Sahl au corps. Un des deux gardiens lui coupe la tête au même moment où un autre le transperce de sa lance. Quelque part Mûsa était Tâlib Ibn Sahl, comme il était aussi Abd al-Samad. Et comme déjà auparavant Abd al-Malik était Mûsa.

Les personnages du conte, redisons-le, ne sont que les différentes positions du même sujet, les figures multiples qu'incarnent tour à tour le sujet depuis la sortie de la mère génitrice jusqu'au retour dans la mère-Terre en passant par les mères amantes 3 que le sujet ne cesse de chercher dans son partenaire.

${ }^{1}$ Lacan, Jacques, Ecrits II, Paris, Seuil, coll. Points, 1971, p. 190.

${ }^{2}$ Pommier Gérard, Le Dénouement d'une analyse, Paris, Point Hors Ligne, 1987, p. 152.

${ }^{3}$ Freud, Essai de psychanalyse appliquée, Paris, Gallimard, 1980, p. 103. 
Il ne fallait donc pas pénétrer dans cette ville. Les multiples plaques en marbre blanc ${ }^{1}$ ou en cuivre était remplies de la même promesse : la mort. La mort qui a frappé les plus courageux et les plus forts, la mort qui n'épargne personne. Car dans l'ombre de la mère se tient le père qui dit non. Mûsa se trouve au noeud de deux relations. Celle avec la mère évoquée par le silence et le désert, celle avec le père évoqué par les écriteaux et le rappel des grandes réalisations du passé. La première faisait naître le désir mais la seconde instituait la loi. Sans le père, la loi ne serait pas, et sans la mère, point de désir.

La Ville de Cuivre n'ignore absolument pas cette tension entre le désir et la loi et leur nécessaire connivence. Au contraire, ils sont signifiés partout. Mais Mûsa fait sienne la loi du père, avant même de pénétrer dans la ville, en apercevant sur la montagne les sept plaques de marbres et en faisant recopier soigneusement toutes les écritures. Il la fait tellement sienne qu'il quitte la ville en fermant la porte comme si rien ne s'était passé (al-amîr Mûsa amarahum an yaghliqûu l-bâba kama kâna) (p. 227). Il la fait sienne au point qu'au terme de la quête, de retour à Damas, il demande au Calife la permission de partir à Jérusalem pour y finir ses jours dans la prière et l'adoration de Dieu (p. 229). Lui, le gouverneur de tout le Maghreb, qui est au sommet de la gloire et de la puissance, renonce subitement à tout comme dans la conscience d'une faute commise et expie le vol, le viol et l'inceste dans la cité symbolique de la vertu et de la loi. Mûsa meurt dans cette ville, anti-thèse de la ville de Cuivre, afin que vive la parole du père et que cesse le désir de la mère.

Shéhérazade, au terme de ce conte, a une fois de plus fait avancer le roi Shahrayâr sur la lente voie de la guérison. En évoquant la peur de la castration, elle permet au roi d'en prendre conscience et ainsi de la dominer. En faisant découvrir au monarque que la ville est comparable au corps d'une femme, il apprend à ne plus y pénétrer par la force et la ruse, sans que la ville se venge. Shahrayâr enfin apprend à faire le deuil de la mère fantasmatique, renvoyant sans

1Freud établir également un lien entre la blancheur et le corps maternel : Cinq psychanalyses, Paris, Puf, 1979, p. 335. 
cesse à la mort, pour mieux s'attacher à celle qui donne la vie et l'espérance.

\section{Bibliographie}

Article Iram de Montgomery-Watt, in E. I., vol. III, Leiden 1975.

Basset, R., Salomon et les djinns rebelles, in Rev. des Trad.pop., Paris, 1888.

Bencheikh, J. D., Les Mille et Une Nuits ou la parole prisonnière, Paris, Gallimard, 1988.

Clouston, Le diable dans une bouteille, in Popular Tales, I, 1887.

Hamori, A., An Allegory from the Arabian Night : the City of Brass, in Bulletin of the School of Oriental and African Studies, 1971, vol. $34, n^{\circ} 1$.

Les cents et une Nuits, trad. Gaudefroy-Demombynes, Paris, Sindbad, 1982.

Miquel, André, La Géographie humaine du monde musulman, vol. II : Les pays de la légende, Paris, Mouton, 1968.

Roth, G., The City of Iram in Ancient Indian Literature and Arabian Nights, in Journal Bihar Res. Soc., 45, 1959.

Tarshûna M., Mi'at laylatin wa layla, Tunis, al-dâr al- ${ }^{\circ}$ arabiyya li-1kitâb, 1979. 